\title{
The bacteria-positive proportion in the disc tissue samples from surgery: a systematic review and meta-analysis
}

\author{
Yucheng Jiao ${ }^{1,2} \cdot$ Yazhou Lin ${ }^{1,2} \cdot$ Yuehuan Zheng $^{3} \cdot$ Ye Yuan $^{4} \cdot$ Zhe Chen $^{1,2} \cdot$ Peng Cao ${ }^{1,2}$
}

Received: 11 September 2018 / Revised: 8 April 2019 / Accepted: 10 June 2019 / Published online: 16 July 2019

(c) The Author(s) 2019

\begin{abstract}
Purpose The role of bacteria, especially Propionibacterium acnes (P. acnes), in human intervertebral disc diseases has raised attention in recent years. However, limited sample size of these studies and diverse bacteria-positive proportion made this topic still controversial. We aimed to review related articles and summarize the bacteria-positive proportion in these studies. Methods We searched the PubMed, Cochrane Library, Embase for related literature from January 2001 to May 2018, and the reference articles were also searched. The random effects or fixed effects meta-analysis was used to pool the overall positive proportion or odds ratio of these studies.

Results We found 16 relevant articles and 2084 cases of the bacteria culture from surgery. Within the 16 included studies, 12 studies' results supported the infection in the discs. The pooled bacterial infection rate was $25.3 \%$. The pooled P. acnes infection rate was $15.5 \%$. The overall pooled P. acnes proportion in bacteria-positive discs was $56.4 \%$. We also found that the presence of bacteria may contribute to the development of Modic change with the odds ratio as 1.27 (95\% CI: 0.44-3.64), but this result is not significant due to heterogeneity, so further study is needed.

Conclusion The existence of bacteria in the intervertebral discs was proved by many studies. However, the variety in sample collecting and culture methods is still obvious and the positive rate also fluctuated within the studies. Standardized and reliable methods should be taken to promote the study in the future.
\end{abstract}

\section{Graphic abstract}

These slides can be retrieved under Electronic Supplementary Material.
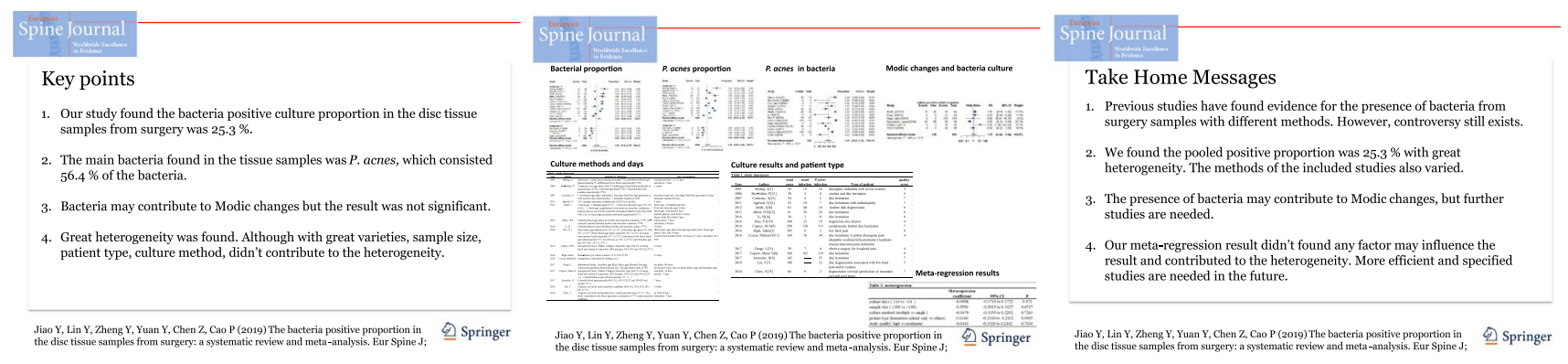

Keywords Intervertebral discs $\cdot$ Bacteria-positive proportion $\cdot$ Meta-analysis

Electronic supplementary material The online version of this article (https://doi.org/10.1007/s00586-019-06062-6) contains supplementary material, which is available to authorized users.
Zhe Chen
drchenzhe@live.com
Peng Cao
dr_caopeng8@163.com

Extended author information available on the last page of the article

\section{Introduction}

After the pioneering study of Stirling [1], the presence of bacteria in the human non-pyogenic intervertebral discs has drawn increasing attention. The potential significance of this finding may change people's understanding of intervertebral disc diseases. Then, Albert's randomized controlled trial 
demonstrated that their antibiotic treatment was more effective for the patients of Modic type 1 changes and chronic low back pain [2]. Their study supported the existence of bacteria and their significant role in intervertebral disc diseases.

There have been an increasing number of studies on this topic over the recent years. Different methods with more cases were used to prove the existence of the bacteria. However, the results of these studies failed to connect the correlation between existence and proportion of the bacteria in human intervertebral discs. The bacterial culture positive rate ranged from $2 \%$ [3] to 53\% [1]. The limited sample size of these studies and the divided opinion of the presence of bacteria made the results insufficient to be applied to clinical decision making. Therefore, a systematic review and metaanalysis of the cultural positive rate could be of importance to serve as a reference for further research.

In this study, we aim to find the bacterial culture positive proportion in non-pyogenic human intervertebral disc samples from surgery and to explain the reasons for the different results.

\section{Methods}

This systematic review was conducted according to the PRISMA statement [4] (see Supplementary Table 1).

\section{Literature search strategy}

We searched the PubMed, Cochrane Library, Embase for studies of intervertebral disc infections published between December 31, 2000 and May 31, 2018. The search was limited to studies in English language. We used the keywords of intervertebral disc, Modic changes, disc herniation, Propionibacterium acnes and infections (see supplementary). We also searched and reviewed the reference of retrieved articles for any potentially relevant studies.

\section{Selection criteria}

Studies were selected according to the following inclusion criteria: (1) during surgery, intervertebral disc samples were collected from patients without artificial implant in the intervertebral discs nor intervertebral discitis before the surgery and (2) the presence of a bacterial culture to test the existence of the bacteria in these samples. Studies without reporting the bacteria culture results were excluded. Case reports, conference presentations, abstracts, expert opinions, reviews and editorials were also excluded. Only the most complete studies would be included if there were series studies with accumulating samples from the same institutions.

\section{Quality assessment}

An 11-item checklist from the Agency for Healthcare Research and Quality (AHRQ) was used to assess the methodological quality of the studies. The item would be given a score of "1" with the answer "Yes" and a score of "0" with the answer "NO" or "UNCLEAR". Article quality will be defined as: low quality $=0-3$; moderate quality $=4-7$; and high quality $=8-11$.

\section{Data extraction}

Data were extracted from texts, figures and tables. Two researchers independently reviewed and extracted data from the articles, specifically, publication year, type of patient, the method of culturing, days of culturing, number of included cases, number of $P$. acnes-positive cultures, number of total bacteria-positive cultures and number of Modic changes. We collected the data about the positive number of the patients or discs when it is mentioned. A consensus discussion was held when there were disagreements about data extraction.

\section{Statistical analysis}

Meta-analysis of proportions was performed for the variable. The analysis was performed by using the meta-package (version 4.9-0) for $\mathrm{R}$ (version 3.3.2). The Freeman-Tukey transformation was used [5]. If the sample size was less than 10, the Arcsine transformation would be used as a replacement. Proportions were combined with random effects models, and the odds ratios were combined with fixed effects model. We used the Wilson method to calculate the $95 \%$ confidence interval (CI). Heterogeneity was evaluated with $I^{2}$. $P$ values of less than 0.05 were defined as statistically significant. Publication bias was tested by Egger's test and Peter's test and funnel plot. A subgroup analysis was performed on the basis of studies supporting or opposing the existence of bacteria. To investigate potential sources of heterogeneity, we performed metaregression with bacteria culture days (by comparing studies with culture period more than 14 days with less 14 days), sample size (by comparing studies of more than 100 samples with smaller studies), culture method (by comparing studies with multiple culture media with single media), patient type (by comparing studies with herniation-related patients with others), study quality (by comparing high-quality studies with moderate-quality studies).

\section{Results}

\section{Search results}

A total of 2882 records were found (see supplementary Figure 1). After the initial screening, we reviewed 25 papers 
in full. Zhou [6], Yuan [7], Yuan [8] are overlapped with Lin [9]. Rollason [10], Aghazadeh [11] are overlapped with Albert [12], Naghmeh Javanshir [13], respectively. After removal of the accumulated reports and exclusion of ineligible reports, 16 studies published between June 2001 and May 2018 with a total of 2084 reported cases were included in the process for pooling the proportion (Table 1).

\section{Study characteristics}

All the included studies reported the existence of P. acnes. Every study reported more than one kind of bacteria except the studies by Javanshir [13] and Lin [9] (See supplementary Table 2). Two studies included more than 300 cases [3, 14]. One study [15] had more than 200 cases, and four studies reported more than 100 cases [9, 13, 16, 17]. All the studies used the bacteria culture, and two studies also detected bacteria DNA in the tissues $[12,15]$. Different culture methods and different culture times were used in these studies (Table 2).

\section{Methodological quality assessment}

The quality of the studies is presented in Table 1. Scores ranged from 4 to 8 . Three included studies were of high quality. The rest of the studies were of moderate quality. Within studies with moderate quality, five studies had a score of 7 which is near to the high quality.

\section{Publication bias}

We assessed all 16 included studies with total case number and cultural positive number. Egger's test did not show risk of publication bias. $(t=0.057, p$ value $=0.9551)$. Peter's test also did not show risk of publication bias. $(t=-1.1756, p$ value $=0.2594$ ). Funnel plot did not show publication bias. (Supplementary Figure 2).

\section{Pooled proportion of disc infection}

From the 16 studies, 2084 cases were included. The pooled proportion of bacteria in the discs was $25.3 \%$ (95\% confidence interval CI, $\left.15.2-37.0 \% ; I^{2}=96.8 \% ; P<0.0001\right)$. Based on the results of the studies supporting or opposing the existence of the bacteria in the intervertebral discs, subgroup analysis was performed. The pooled proportion of the bacteria in the discs from the studies that supported the existence of bacteria in intervertebral discs was $33.7 \%$ (95\% confidence interval CI, 26.4-41.4\%). The pooled proportion of the bacteria in discs from the other group studies was $5.0 \%$ (95\% confidence interval CI, 0.7-11.9\%) (Fig. 1).

\section{Pooled proportion of $P$. acnes infection in disc samples}

From these 16 articles, the pooled proportion of $P$. acnespositive proportion was $15.5 \%$ (95\% confidence interval CI, 7.7-25.3\%, $\left.I^{2}=96.5 \%, P<0.0001\right)$. From the studies that support the existence of the bacteria, the pooled

Table 1 Study characteristics

\begin{tabular}{|c|c|c|c|c|c|c|}
\hline Year & Author & Total cases & Total infection & $\begin{array}{l}\text { P. acnes } \\
\text { infection }\end{array}$ & Type of patient & Quality score \\
\hline 2001 & Stirling [1] & 36 & 19 & 16 & Discogenic radiculitis with severe sciatica & 5 \\
\hline 2006 & Ben-Galim [26] & 30 & 2 & 0 & Sciatica and disc herniation & 5 \\
\hline 2007 & Carricajo [27] & 54 & 4 & 2 & Disc herniation & 7 \\
\hline 2011 & Agarwal [44] & 52 & 10 & 7 & Disc herniation with radiculopathy & 7 \\
\hline 2012 & Arndt [33] & 83 & 40 & 18 & Lumbar disc degeneration & 6 \\
\hline 2013 & Albert [12] & 61 & 28 & 24 & Disc herniation & 8 \\
\hline 2016 & $\operatorname{Li}[21]$ & 30 & 3 & 0 & Disc herniation & 6 \\
\hline 2016 & Rao [16] & 168 & 33 & 19 & Degenerate disc disease & 4 \\
\hline 2016 & Capoor [15] & 290 & 130 & 115 & Symptomatic lumbar disc herniation & 7 \\
\hline 2016 & Rigal [3] & 385 & 6 & 2 & Low back pain & 8 \\
\hline 2016 & Coscia [17] & 169 & 76 & 34 & $\begin{array}{l}\text { Disc herniation/lumbar discogenic pain idiopathic scoliosis/ } \\
\text { Scheuermann's kyphosis trauma/neuromuscular deformity }\end{array}$ & 4 \\
\hline 2017 & Drago [34] & 39 & 7 & 4 & Elective surgery for low back pain & 4 \\
\hline 2017 & Capoor [14] & 368 & 162 & 119 & Disc herniation & 8 \\
\hline 2017 & Javanshir [13] & 145 & - & 55 & Disc herniation & 7 \\
\hline 2018 & Lin [9] & 108 & - & 23 & Disc degeneration associated with low back pain and/or sciatica & 5 \\
\hline 2018 & Chen $[30]$ & 66 & 9 & 2 & $\begin{array}{l}\text { Degenerative cervical spondylosis or traumatic cervical cord } \\
\text { injury }\end{array}$ & 7 \\
\hline
\end{tabular}


Table 2 Study characteristics

\begin{tabular}{|c|c|c|c|}
\hline Year & Author & Method of culturing & Days of culturing \\
\hline 2001 & Stirling, A & $\begin{array}{l}\text { Robertson's cooked meat enrichment broth }\left(37^{\circ} \mathrm{C}\right) \text {; sub- } \\
\text { culture at blood agar plates containing } 7 \% \text { defibrinated } \\
\text { horse blood anaerobically }\left(37^{\circ} \mathrm{C}\right)\end{array}$ & $\begin{array}{l}\text { Enrichment broth: } 2,7,21 \text { days } \\
\text { Subculture: } 7 \text { days }\end{array}$ \\
\hline 2006 & Ben-Galim, P & $\begin{array}{l}2 \text { Trypticase soy agar plates with } 5 \% \text { defibrinated sheep } \\
\text { blood aerobically or anaerobically }\left(37^{\circ} \mathrm{C}\right) ; 1 \text { choco- } \\
\text { late agar plate }\left(37^{\circ} \mathrm{C}\right) ; 1 \text { liquid thioglycolate medium } \\
\text { anaerobically }\left(37^{\circ} \mathrm{C}\right)\end{array}$ & 2 weeks \\
\hline 2007 & Carricajo, A & $\begin{array}{l}1 \text { Horse blood agar plate aerobically; } 2 \text { chocolate Poly- } \\
\text { Vitex agar plates (one in } \mathrm{CO} 2 \text { and the other anaerobi- } \\
\text { cally); } 1 \text { Schaedler medium at } 36^{\circ} \mathrm{C}\end{array}$ & $\begin{array}{l}\text { Horse blood agar and chocolate PolyVitex agar plates: } \\
10 \text { days } \\
\text { Schaedler medium: } 20 \text { days }\end{array}$ \\
\hline 2011 & Agarwal, V & $\begin{array}{l}35^{\circ} \mathrm{C} \text {, standard anaerobic condition, and } 5 \% \mathrm{CO}_{2} \text { for } \\
\text { aerobes }\end{array}$ & 5 days \\
\hline 2012 & Arndt, J & $\begin{array}{l}1 \text { Blood agar, } 1 \text { Drigalski agar }\left(37^{\circ} \mathrm{C}\right) ; 1 \text { PolyVitex } \\
\text { chocolate agar }\left(37^{\circ} \mathrm{C}, 5 \% \mathrm{CO}_{2}\right) ; 1 \text { blood agar sup- } \\
\text { plemented with hemin (in anaerobic conditions); } 1 \\
\text { peptone glucose yeast broth (anaerobic atmosphere); } \\
\text { BACTEC Peds Plus bottle with } 2 \mathrm{~mL} \text { of fructooligo- } \\
\text { saccharide nutritional supplement }\left(35^{\circ} \mathrm{C}\right)\end{array}$ & $\begin{array}{l}\text { Blood agar, Drigalski agar: } 24 \mathrm{~h} \\
\text { PolyVitex chocolate agar } 4 \text { days } \\
\text { Blood agar with hemin: } 5 \text { days } \\
\text { Peptone glucose yeast broth: } 10 \text { days } \\
\text { BACTEC Peds Plus bottle: } 7 \text { days }\end{array}$ \\
\hline 2013 & Albert, H B & $\begin{array}{l}\text { Columbia blood agar plates (in aerobic and anaerobic } \\
\text { conditions } 37^{\circ} \mathrm{C} \text { ); subcultured Columbia blood (in } \\
\text { aerobic and anaerobic conditions } 37^{\circ} \mathrm{C} \text { ) }\end{array}$ & $\begin{array}{l}\text { Initial culture: } 7 \text { days; } \\
\text { Subculture: } 24 \mathrm{~h}\end{array}$ \\
\hline 2016 & $\mathrm{Li}, \mathrm{B}$ & $\begin{array}{l}\text { Columbia Blood Culture Medium (aerobic and anaerobic } \\
\text { culture } 37^{\circ} \mathrm{C} \text { ) }\end{array}$ & 10 days \\
\hline 2016 & Rao, P J & $\begin{array}{l}\text { MacConkey agar plate (aerobic } 35^{\circ} \mathrm{C} \text { to } 37^{\circ} \mathrm{C} \text { ); choco- } \\
\text { late agar plate }\left(5 \% \mathrm{CO} 235^{\circ} \mathrm{C} \text { to } 37^{\circ} \mathrm{C} \text { ); horse blood }\right. \\
\text { agar plates (anaerobic } 35^{\circ} \mathrm{C} \text { to } 37^{\circ} \mathrm{C} \text { ); cooked meat } \\
\text { medium broth (anaerobic } 35^{\circ} \mathrm{C} \text { to } 37^{\circ} \mathrm{C} \text { ) subcultured } \\
\text { with horse blood agar plate (anaerobic, } 37^{\circ} \mathrm{C} \text { for } 48 \mathrm{~h} \\
\text { or } 35^{\circ} \mathrm{C} \text { to } 37^{\circ} \mathrm{C} \text { ) and chocolate agar plat }(5 \% \mathrm{CO} \text {, } \\
35^{\circ} \mathrm{C} \text { to } 37^{\circ} \mathrm{C} \text { ) }\end{array}$ & $\begin{array}{l}\text { MacConkey agar plate, chocolate agar plate, horse blood } \\
\text { agar plates: } 7 \text { days and } 14 \text { days } \\
\text { Cooked meat medium broth: } 48 \text { h or } 7 \text { days subculture for } \\
7 \text { days }\end{array}$ \\
\hline 2016 & Capoor, M N & $\begin{array}{l}\text { homogenized tissue, Wilkins Chalgren Anaerobic Agar } \\
\text { with } 7 \% \text { of sheep blood and vitamin } \mathrm{K} \text { (anaerobic, } 80 \% \\
\text { nitrogen, } 10 \% \mathrm{CO} 2 \text { and } 10 \% \mathrm{H} 2,37^{\circ} \mathrm{C} \text { ) }\end{array}$ & 14 days \\
\hline 2016 & Rigal, Julien & Brain-heart-type culture medium $\left(5 \% \mathrm{CO}_{2}, 37^{\circ} \mathrm{C}\right)$ & 15 days \\
\hline 2016 & Coscia, Michael F & Comparable to described by Stirling et al. [1] & \\
\hline 2017 & Drago, L & $\begin{array}{l}\text { Dithiothreitol eluate, chocolate agar, MacConkey agar, } \\
\text { Mannitol Salt agar; Sabouraud agar; brain heart infu- } \\
\text { sion and Thioglycollate broths }\left(37^{\circ} \mathrm{C}\right)\end{array}$ & $\begin{array}{l}\text { For plates: } 48 \mathrm{~h} \\
\text { For broths: } 15 \text { days then on sheep blood agar and Schae- } \\
\text { dler agar }\end{array}$ \\
\hline 2017 & Capoor, Manu N & $\begin{array}{l}\text { Homogenized tissue, Wilkins Chalgren Anaerobic Agar } \\
\text { with } 7 \% \text { of sheep blood and vitamin } \mathrm{K} \text { (anaerobic, } 80 \% \\
\text { nitrogen, } 10 \% \mathrm{CO}_{2} \text { and } 10 \% \mathrm{H} 2,37^{\circ} \mathrm{C} \text { ), Columbia } \\
\text { blood agar (Oxoid) (aerobic, } 37^{\circ} \mathrm{C} \text { ) }\end{array}$ & $\begin{array}{l}\text { Anaerobic: } 14 \text { days } \\
\text { Aerobic: } 7 \text { days }\end{array}$ \\
\hline 2017 & Javanshir, $\mathrm{N}$ & $\begin{array}{l}\text { Columbia blood agar (anaerobic: } 80 \% \mathrm{~N}_{2}, 10 \% \mathrm{CO}_{2}, 37 \\
\text { and } 10 \% \mathrm{H} 2 \text { and aerobic, } 37^{\circ} \mathrm{C} \text { ) }\end{array}$ & 7 days \\
\hline 2018 & Lin, $Y$ & $\begin{array}{l}\text { Tryptone soy broth under anaerobic conditions }\left(80 \% \mathrm{~N}_{2} \text {, }\right. \\
\left.10 \% \mathrm{CO}_{2}, 10 \% \mathrm{H} 2,37^{\circ} \mathrm{C}\right)\end{array}$ & 14 days \\
\hline 2018 & Chen, Y. & $\begin{array}{l}\text { Tryptone soy broth and incubated in a sealed anaerobic } \\
\text { bag at } 37^{\circ} \mathrm{C} \text {. The broth subcultured onto blood agar } \\
\text { plates, incubated at } 37^{\circ} \mathrm{C} \text { under anaerobic conditions }\end{array}$ & $\begin{array}{l}\text { In TSB: } 14 \text { days } \\
\text { Subculture: } 7 \text { days }\end{array}$ \\
\hline
\end{tabular}

proportion of the $P$. acnes infection in discs was $23.3 \%$ (95\% confidence interval CI, 16.3-31.2\%). For the four studies that disputed the existence of the bacteria, the pooled proportion was $0.3 \%$ (95\% confidence interval CI, 0.0-1.5\%) (Fig. 2). 


\begin{tabular}{|c|c|c|c|c|c|c|c|}
\hline Study & Events & Total & & & Proportion & $95 \%-\mathrm{Cl}$ & Weight \\
\hline subgroup $=1$ & & & & & & & \\
\hline Stirling, $A(2001)$ & 19 & 36 & & 母 & 0.53 & {$[0.37 ; 0.68]$} & $5.9 \%$ \\
\hline Agarwal, V(2011) & 10 & 52 & 田 & $\vdots$ & 0.19 & {$[0.11 ; 0.32]$} & $6.1 \%$ \\
\hline Arndt, J(2012) & 40 & 83 & & —- & 0.48 & {$[0.38 ; 0.59]$} & $6.3 \%$ \\
\hline Albert, H B(2013) & 28 & 61 & & —- & 0.46 & {$[0.34 ; 0.58]$} & $6.2 \%$ \\
\hline Rao, P J(2016) & 33 & 168 & 世 & & 0.20 & {$[0.14 ; 0.26]$} & $6.5 \%$ \\
\hline Capoor, M N(2016) & 130 & 290 & & \# & 0.45 & {$[0.39 ; 0.51]$} & $6.5 \%$ \\
\hline Coscia, Michael F(2016) & 76 & 169 & & — & 0.45 & {$[0.38 ; 0.52]$} & $6.5 \%$ \\
\hline Drago, L(2017) & 7 & 39 & 田 & : & 0.18 & {$[0.09 ; 0.33]$} & $6.0 \%$ \\
\hline Capoor, Manu N.(2017) & 162 & 368 & & 母 & 0.44 & {$[0.39 ; 0.49]$} & $6.5 \%$ \\
\hline Javanshir, N. (2017) & 55 & 145 & & \#- & 0.38 & {$[0.30 ; 0.46]$} & $6.4 \%$ \\
\hline Lin, Y. (2018) & 23 & 108 & 田 & & 0.21 & {$[0.15 ; 0.30]$} & $6.4 \%$ \\
\hline Chen, Y.(2018) & 9 & 66 & 曰- & & 0.14 & {$[0.07 ; 0.24]$} & $6.2 \%$ \\
\hline $\begin{array}{l}\text { Random effects model } \\
\text { Heterogeneity: } I^{2}=89 \%, p<\end{array}$ & & 1585 & & $\infty$ & 0.34 & {$[0.26 ; 0.41]$} & $75.6 \%$ \\
\hline subgroup $=2$ & & & & & & & \\
\hline Ben-Galim, P.(2006) & 2 & 30 & \# & & 0.07 & {$[0.02 ; 0.21]$} & $5.8 \%$ \\
\hline Carricajo, A.(2007) & 4 & 54 & \#- & & 0.07 & {$[0.03 ; 0.18]$} & $6.2 \%$ \\
\hline Li, B(2016) & 3 & 30 & 世 & & 0.10 & {$[0.03 ; 0.26]$} & $5.8 \%$ \\
\hline Rigal, Julien(2016) & 6 & 385 & 田 & & 0.02 & {$[0.01 ; 0.03]$} & $6.5 \%$ \\
\hline $\begin{array}{l}\text { Random effects model } \\
\text { Heterogeneity: } I^{2}=72 \%, p=\end{array}$ & 0.01 & 499 & $\infty$ & & 0.05 & {$[0.01 ; 0.12]$} & $24.4 \%$ \\
\hline Random effects $\mathbf{n}$ & & 2084 & & & 0.25 & {$[0.15 ; 0.37]$} & $100.0 \%$ \\
\hline & & & 0.1 & 30 & & & \\
\hline
\end{tabular}

Fig. 1 Forest plot for pooled proportion of disc-positive culture. Subgroup 1 represents studies that support the existence of bacterial infection. Subgroup 2 represents studies that do not support the existence of bacterial infection. CI: confidence interval

\section{Pooled proportion of P. acnes infection in the bacteria-positive discs}

The most common bacteria are the $P$. acnes. The pooled proportion of $P$. acnes in the infected discs was $56.4 \%(95 \%$ confidence interval CI, $\left.42.3-70.0 \%, I^{2}=87.2 \%, P<0.0001\right)$ (Fig. 3).

\section{Comparison of Modic change between positive cultures and negative cultures}

Among the included papers that were analysed for the infection proportion of $P$. acnes, we found Arndt [33], Rigal [3], Drago [34], Chen [30] analysed the presence of Modic changes. The papers of Aghazadeh [11] and Zhou [6] which were excluded due to overlapped data in analysis of infection proportion were also included for assessment of Modic changes (Supplemental Table 3). Overall, the percentages of Modic changes in positive cultures versus non-positive cultures among the six papers were: 77.5 versus $65 \%, 55.6$ versus $38.2 \%, 100$ versus $93.7 \%, 61.8$ versus $86.5 \%, 85.7$ versus $31.3 \%, 44.4$ versus $46.4 \%$, respectively. Meta-analysis found the pooled odds ratio to be 1.27 (95\% CI: $\left.0.44-3.64, I^{2}=68 \%, p<0.01\right)$ with random effects model; however, heterogeneity was significant (Fig. 4). Thus, we did the sensitivity analysis and found the result of Aghazadeh [11] was different from others which may be the main source of heterogeneity, even though they also found significant relationship between $P$. acnes-positive samples and presence of MCs in the sample. We re-evaluated meta-analysis without that article and got the reformative result with fixed effects model as 1.97 (95\% CI: $1.04-3.73, I^{2}=4 \%, p=0.39$, Supplemental Figure 3).

\section{Heterogeneity analysis}

The pooled proportion of positive rates were significantly different between subgroups that support or dispute the existence of infection. However, meta-regression results showed that the difference of bacteria culture days, multiculture methods, sample sizes, disc herniation or study quality did not contribute to heterogeneity (Table 3 ). 


\begin{tabular}{|c|c|c|c|c|c|c|c|}
\hline Study & Events & Total & & & ortion & $95 \%-\mathrm{Cl}$ & Weight \\
\hline \multicolumn{8}{|l|}{ subgroup = 1} \\
\hline Stirling, $A(2001)$ & 16 & 36 & & $\mp$ & 0.44 & {$[0.30 ; 0.60]$} & $5.9 \%$ \\
\hline Agarwal, V(2011) & 7 & 52 & & & 0.13 & {$[0.07 ; 0.25]$} & $6.1 \%$ \\
\hline Arndt, J(2012) & 18 & 83 & & — & 0.22 & {$[0.14 ; 0.32]$} & $6.3 \%$ \\
\hline Albert, H B(2013) & 24 & 61 & & — & 0.39 & {$[0.28 ; 0.52]$} & $6.2 \%$ \\
\hline Rao, P J(2016) & 19 & 168 & \# & & 0.11 & {$[0.07 ; 0.17]$} & $6.5 \%$ \\
\hline Capoor, M N(2016) & 115 & 290 & & \# & 0.40 & {$[0.34 ; 0.45]$} & $6.5 \%$ \\
\hline Coscia, Michael F(2016) & 34 & 169 & & - & 0.20 & {$[0.15 ; 0.27]$} & $6.5 \%$ \\
\hline Drago, L(2017) & 4 & 39 & 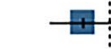 & & 0.10 & {$[0.04 ; 0.24]$} & $6.0 \%$ \\
\hline Capoor, Manu N.(2017) & 119 & 368 & & 母 & 0.32 & {$[0.28 ; 0.37]$} & $6.6 \%$ \\
\hline Javanshir, N. (2017) & 55 & 145 & & 世 & 0.38 & {$[0.30 ; 0.46]$} & $6.5 \%$ \\
\hline Lin, $Y .(2018)$ & 23 & 108 & & & 0.21 & {$[0.15 ; 0.30]$} & $6.4 \%$ \\
\hline Chen, Y.(2018) & 2 & 66 & 田 & & 0.03 & {$[0.01 ; 0.10]$} & $6.2 \%$ \\
\hline $\begin{array}{l}\text { Random effects model } \\
\text { Heterogeneity: } I^{2}=91 \%, p<0\end{array}$ & 0.01 & 1585 & & $\infty$ & 0.23 & {$[0.16 ; 0.31]$} & $75.7 \%$ \\
\hline \multicolumn{8}{|l|}{ subgroup $=2$} \\
\hline Ben-Galim, P.(2006) & 0 & 30 & ๒- & & 0.00 & {$[0.00 ; 0.11]$} & $5.8 \%$ \\
\hline Carricajo, A.(2007) & 2 & 54 & \#- & & 0.04 & {$[0.01 ;$} & $6.1 \%$ \\
\hline $\mathrm{Li}, \mathrm{B}(2016)$ & 0 & 30 & E- & & 0.00 & {$[0.00 ; 0.11]$} & $5.8 \%$ \\
\hline Rigal, Julien(2016) & 2 & 385 & 田 & & 0.01 & {$[0.00 ; 0.02]$} & $6.6 \%$ \\
\hline \multicolumn{8}{|c|}{ Heterogeneity: $I^{2}=12 \%, p=0.33$} \\
\hline \multirow{2}{*}{\multicolumn{2}{|c|}{$\begin{array}{l}\text { Random effects model } \\
\text { Heterogeneity: } I^{2}=97 \%, p<0.01\end{array}$}} & 208 & & & 0.16 & {$[0.08 ; 0.25]$} & $100.0 \%$ \\
\hline & & & & 20 & & & \\
\hline
\end{tabular}

Fig. 2 Forest plot of pooled proportion of P. acnes-positive culture in disc samples. Subgroup 1 represents studies that support the existence of bacterial infection. Subgroup 2 represents studies that do not support the existence of bacterial infection. CI: confidence interval

\begin{tabular}{|c|c|c|c|c|c|c|}
\hline Study & Events & Total & & rtion & $95 \%-\mathrm{Cl}$ & Weight \\
\hline Stirling, $A(2001)$ & 16 & 19 & 母 & 0.84 & {$[0.62 ; 0.94]$} & $8.1 \%$ \\
\hline Ben-Galim, P.(2006) & 0 & 2 & $\mathbb{E}$ & 0.00 & {$[0.00 ; 0.66]$} & $3.0 \%$ \\
\hline Carricajo, A.(2007) & 2 & 4 & —贯: & 0.50 & {$[0.15 ; 0.85]$} & $4.6 \%$ \\
\hline Agarwal, V(2011) & 7 & 10 & 世 & 0.70 & {$[0.40 ; 0.89]$} & $6.8 \%$ \\
\hline Arndt, $\mathrm{J}(2012)$ & 18 & 40 & 回 & 0.45 & {$[0.31 ; 0.60]$} & $9.0 \%$ \\
\hline Albert, H B(2013) & 24 & 28 & 世 & 0.86 & {$[0.69 ; 0.94]$} & $8.6 \%$ \\
\hline Li, B(2016) & 0 & 3 & $\mathbb{E}$ & 0.00 & {$[0.00 ; 0.56]$} & $3.9 \%$ \\
\hline Rao, P J(2016) & 19 & 33 & 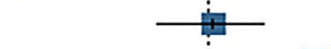 & 0.58 & {$[0.41 ; 0.73]$} & $8.8 \%$ \\
\hline Capoor, M N(2016) & 115 & 130 & 母 & 0.88 & {$[0.82 ; 0.93]$} & $9.7 \%$ \\
\hline Rigal, Julien(2016) & 2 & 6 & 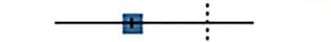 & 0.33 & {$[0.10 ; 0.70]$} & $5.6 \%$ \\
\hline Coscia, Michael F(2016) & 34 & 76 & 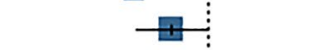 & 0.45 & {$[0.34 ; 0.56]$} & $9.5 \%$ \\
\hline Drago, L(2017) & 4 & 7 & & 0.57 & {$[0.25 ; 0.84]$} & $6.0 \%$ \\
\hline Capoor, Manu N.(2017) & 119 & 162 & 丹 & 0.73 & {$[0.66 ; 0.80]$} & $9.8 \%$ \\
\hline Chen, Y.(2018) & 2 & 9 & —专 & 0.22 & {$[0.06 ; 0.55]$} & $6.6 \%$ \\
\hline Random effects model & & 529 & 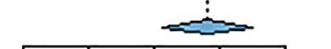 & 0.56 & {$[0.42 ; 0.70]$} & $100.0 \%$ \\
\hline Heterogeneity: $I^{2}=87 \%, p$ & & & $\Gamma 1$ & & & \\
\hline & & & $\begin{array}{lllll}0 & 0.2 & 0.4 & 0.6 & 0.8\end{array}$ & & & \\
\hline
\end{tabular}

Fig. 3 Forest plot of pooled proportion of P. acnes-positive culture in the bacteria-positive discs 


\begin{tabular}{|c|c|c|c|c|c|c|c|c|}
\hline \multirow[b]{2}{*}{ Study } & \multicolumn{4}{|c|}{ cultrue-positive culture-negative } & \multirow[b]{2}{*}{ Odds Ratio } & \multirow[b]{2}{*}{ OR } & \multirow[b]{2}{*}{$95 \%-\mathrm{Cl}$} & \multirow[b]{2}{*}{ Weight } \\
\hline & Events & Total & Events & Total & & & & \\
\hline Arndt, J(2012) & 31 & 40 & 26 & 40 & & 1.85 & {$[0.69 ; 4.97]$} & $21.6 \%$ \\
\hline Zhou, Z(2015) & 5 & 9 & 13 & 34 & & 2.02 & {$[0.46 ; 8.92]$} & $17.4 \%$ \\
\hline Rigal, Julien(2016) & 6 & 6 & 355 & 379 & & 0.90 & {$[0.05 ; 16.37]$} & $8.8 \%$ \\
\hline Aghazadeh, Javad(2016) & 42 & 68 & 45 & 52 & 田 & 0.25 & {$[0.10 ; 0.64]$} & $22.1 \%$ \\
\hline Drago, L.(2017) & 6 & 7 & 10 & 32 & 五 & -13.20 & {$[1.40 ; 124.63]$} & $12.1 \%$ \\
\hline Chen,Y.(2018) & 4 & 9 & 26 & 56 & & 0.92 & {$[0.22 ; 3.80]$} & $18.0 \%$ \\
\hline \multirow{2}{*}{\multicolumn{2}{|c|}{$\begin{array}{l}\text { Random effects model } \\
\text { Heterogeneity: } I^{2}=68 \%, p<0.01\end{array}$}} & 139 & & 593 & $+\infty$ & 1.27 & {$[0.44 ; 3.64]$} & $100.0 \%$ \\
\hline & & & & & 10 & 00 & & \\
\hline
\end{tabular}

Fig. 4 Forest plot of Modic changes between culture-positive samples and culture-negative samples

Table 3 Meta-regression

\begin{tabular}{lccl}
\hline & $\begin{array}{l}\text { Metaregression } \\
\text { coefficient }\end{array}$ & $95 \%$ CI & $P$ \\
\hline Culture days $(\geq 14$ vs $<14)$ & -0.0998 & -0.3719 to 0.1722 & 0.472 \\
Sample size $(\geq 100$ vs $<100)$ & 0.0556 & -0.2015 to 0.3127 & 0.6717 \\
Culture method (multiple vs single) & -0.0479 & -0.3159 to 0.2202 & 0.7263 \\
Patient type (herniation related only vs others) & 0.0146 & -0.2140 to 0.2432 & 0.9005 \\
Study quality(high vs moderate) & -0.0443 & -0.3328 to 0.2442 & 0.7634 \\
\hline
\end{tabular}

\section{Discussion}

Our study found that the pooled proportion of the positive bacterial culture was $25.3 \%$ from the studies published between January 2001 and May 2018. Among the bacteria, $56.4 \%$ were $P$. acnes. And the pooled infection of P. acnes in these studies was $15.5 \%$. This demonstrated that $P$. acnes was the major bacterium in the culture-positive discs. The odds ratio of Modic change in culture-positive samples is 1.27 (95\% CI: $0.44-3.64, I^{2}=68 \%, p<0.01$ ). This indicated that the presence of bacteria may contribute to the development of Modic changes; however, the result is not significant.

Propionibacterium acnes (P. acnes) is an aerotolerant, anaerobic, Gram-positive bacterium known as a skin commensal. P. acnes is also an opportunistic pathogen that causes implant-related infection as well as infections of bones and joints $[18,19]$. The relatively avascular space of the intervertebral disc is an ideal environment for $P$. acnes to survive [20]. Animal models also proved that the disc environment was optimal for the bacteria [21] and inoculation of $P$. acnes can cause degenerative-like changes in the intervertebral discs $[9,21-25]$. This may account for the fact that $P$. acnes is the major bacteria in the positive samples and suggests that $P$. acnes could be the culprit of some intervertebral disc diseases.

However, the culture-positive proportion varied within studies and some studies did not support the existence of bacteria in the intervertebral discs $[3,21,26,27]$. The heterogeneity was significant. Our study also demonstrated that the significantly different culture-positive proportions between groups support or oppose the existence of bacteria. The main controversy is whether positive results are related to contamination. P. acnes, a common skin commensal, is considered to be related to orthopaedic surgery contamination $[28,29]$. To distinguish contamination from the real infection, some studies used muscle or other related tissue biopsy cultures to serve as a control group $[6,11,27,30]$. However, most studies did not set control groups to eliminate the possibility of contamination. Capoor's [15] study also suggested the significant bacterial counts as distinction from the contamination.

On the other hand, $P$. acnes culture requires a relatively strict anaerobic condition. It grows slower than other anaerobic bacteria [31], and the efficiency of culture methods is important. Suan's study suggested that more than a 13-day culture period and both anaerobic and aerobic culture methods were needed for P. acnes recovery [32]. Among our included studies, adequate culture time period up to 14 days or more is used in many of the studies $[1,3,9,15,16,26$, 27, 30]. Multiple culture mediums for the tissues were also used to prevent false-negative result [16, 26, 27, 33, 34]. However, according to the meta-regression results, these differences among studies did not contribute to the heterogeneity of the pooled proportions. Attention should still be paid to the culture method. Additionally, Capoor et al. [15] suggested homogenizing the disc samples before culture, 
concerning the biofilm form of $P$. acnes, would improve the culture efficiency.

Although the culture result is controversial, many other methods like HE and modified Brown-Brenn staining [7], confocal scanning laser microscopy [14], fluorescence in situ hybridization [35], liquid chromatography-tandem mass spectrometry (LC-MS/MS) [36] and PCR assay [12, 15, 36, 37] have provided the evidence for the presence of bacteria. Studies have also demonstrated the differences between genotypes of $P$. acnes from intervertebral discs and skin. This is evidence that the culture-positive results are not just the contamination from surgery $[10,38]$.

Although study has found intervertebral herniation is associated with positive bacterial culture [17], our metaregression result did not find that patient type, especially herniation, contributed to the heterogeneity. This may be explained by the fact that the presence of bacteria can cause the degenerate changes and its related symptoms not just herniation. However, we cannot ignore that the great variety of included studies and the limited study numbers prevent further analysis of the possible correlation between development of low-virulent infection and its corresponding intervertebral disc diseases and symptoms. Previous systematic reviews had indicated moderate evidence for the relationship between bacteria presence and low back pain with herniation, and Modic change associated with disc herniation [39] or disc degeneration [40]. In the included studies, the highest culture-positive proportions were from studies of severe sciatica patients with confirmed discogenic radiculitis [1], confirmed herniation and its related symptoms and signs $[12,13,15]$. However, with these same kinds of patients, studies also reported cultural results did not support the infection in discs $[26,27]$. In order to further investigate the correlation between the development of infection and the type of degeneration, more studies with specified group patients or control groups are needed in the future.

Great interests have been aroused in the field about the relation between Modic change and disc infection. Many researches have been done [2, 3, 12, 23-25, 34, 41, 42]. Our meta-analysis result indicated that the presence of bacteria may contribute to the development of Modic changes but this result is not significant, with the result of 1.27 (95\% CI: 0.44-3.640). The sensitivity analysis found this result is significant after exclusion of the paper of Aghazadeh [11] which contributes main heterogeneity to this result. The reformative result was 1.97 (95\% CI: 1.04-3.73). In Aghazadeh, Javad's study, their culture period is 7 days which is the lowest. The other studies included in odds ratio meta-analysis all have at least 10-day culture periods. Although their study also found the relationship between Modic change and the presence of $P$. acnes, we suppose that longer culture period may make this meta-analysis result more significant. And further study should be done to explore this question.
Because of the individual differences, large sample size is still needed to ensure the clinical feature of this non-pyogenic infection in the future. To fulfil this, a sensitive, reliable, practical or noninvasive clinical test method is required. Stirling's [1] study introduced a serological test to diagnose deep-seated infection by detecting extracellular antigen from bacteria. Magnitsky's [43] study introduced a noninvasive method to detect the existence of $P$. acnes in intervertebral discs in an animal model by NMR spectroscopy. These types of technologies may help in future clinical study.

There are some limitations to the study. First, the heterogeneity between studies is significant. The variety in methodology may contribute to it but the limited number and the variety of included studies prevent further analysis. Secondly, most of the studies were in a moderate quality and lack of culture control group makes the positive results less convincing. Thus, the results may have moderate contribution to the literature due to the heterogeneity of the articles.

Although an increasing number of studies and evidences demonstrate the existence of the bacteria in the intervertebral discs, the proportion of bacteria in discs still varied from studies. Our study performed a systematic review of the previous study and provided a positive cultural proportion for reference. In order to confirm the results, more cases should be done with efficient method of culture techniques, control groups, specified patients' types, advanced test technologies and so on.

Funding This work was supported by grants from the National Natural Science fund, China (NSFC No. 81702188, No. 81874021).

\section{Compliance with ethical standards}

Conflict of interest The authors declare that they have no competing interests.

Open Access This article is distributed under the terms of the Creative Commons Attribution 4.0 International License (http://creativeco mmons.org/licenses/by/4.0/), which permits unrestricted use, distribution, and reproduction in any medium, provided you give appropriate credit to the original author(s) and the source, provide a link to the Creative Commons license, and indicate if changes were made.

\section{References}

1. Stirling A, Worthington T, Rafiq M, Lambert PA, Elliott TS (2001) Association between sciatica and Propionibacterium acnes. Lancet 357:2024-2025. https://doi.org/10.1016/s0140 $-6736(00) 05109-6$

2. Albert HB, Sorensen JS, Christensen BS, Manniche C (2013) Antibiotic treatment in patients with chronic low back pain and vertebral bone edema (Modic type 1 changes): a double-blind randomized clinical controlled trial of efficacy. Eur Spine J 22:697707. https://doi.org/10.1007/s00586-013-2675-y 
3. Rigal J, Thelen T, Byrne F, Cogniet A, Boissière L, Aunoble S, Le Huec J-C (2016) Prospective study using anterior approach did not show association between Modic 1 changes and low grade infection in lumbar spine. Eur Spine J 25:1000-1005

4. Moher D, Liberati A, Tetzlaff J, Altman DG (2009) Preferred reporting items for systematic reviews and meta-analyses: the PRISMA statement. PLoS Med 6:1-6

5. Barendregt JJ, Doi SA, Lee YY, Norman RE, Vos T (2013) Metaanalysis of prevalence. J Epidemiol Community Health 67:974978. https://doi.org/10.1136/jech-2013-203104

6. Zhou Z, Chen Z, Zheng Y, Cao P, Liang Y, Zhang X, Wu W, Xiao J, Qiu S (2015) Relationship between annular tear and presence of Propionibacterium acnes in lumbar intervertebral disc. Eur Spine J 24:2496-2502. https://doi.org/10.1007/s00586-015-4180-y

7. Yuan Y, Zhou Z, Jiao Y, Li C, Zheng Y, Lin Y, Xiao J, Chen Z, Cao P (2017) Histological Identification of Propionibacterium acnes in nonpyogenic degenerated intervertebral discs. Biomed Res Int 2017:6192935. https://doi.org/10.1155/2017/6192935

8. Yuan Y, Chen Y, Zhou Z, Jiao Y, Li C, Zheng Y, Lin Y, Xiao J, Chen Z, Cao P (2017) Association between chronic inflammation and latent infection of Propionibacterium acnes in non-pyogenic degenerated intervertebral discs: a pilot study. Eur Spine J. https ://doi.org/10.1007/s00586-017-5363-5

9. Lin Y, Jiao Y, Yuan Y, Zhou Z, Zheng Y, Xiao J, Li C, Chen Z, Cao P (2018) Propionibacterium acnes induces intervertebral disc degeneration by promoting nucleus pulposus cell apoptosis via the TLR2/JNK/mitochondrial-mediated pathway. Emerg Microbes Infect 7:1. https://doi.org/10.1038/s41426-017-0002-0

10. Rollason J, McDowell A, Albert HB, Barnard E, Worthington T, Hilton AC, Vernallis A, Patrick S, Elliott T, Lambert P (2013) Genotypic and antimicrobial characterisation of Propionibacterium acnes isolates from surgically excised lumbar disc herniations. BioMed Res Int 2013:1-7

11. Aghazadeh J, Salehpour F, Ziaeii E, Javanshir N, Samadi A, Sadeghi J, Mirzaei F, Naseri Alavi SA (2017) Modic changes in the adjacent vertebrae due to disc material infection with Propionibacterium acnes in patients with lumbar disc herniation. Eur Spine J. 26:3129-3134

12. Albert HB, Lambert P, Rollason J, Sorensen JS, Worthington T, Pedersen MB, Norgaard HS, Vernallis A, Busch F, Manniche C, Elliott T (2013) Does nuclear tissue infected with bacteria following disc herniations lead to Modic changes in the adjacent vertebrae? Eur Spine J 22:690-696. https://doi.org/10.1007/s0058 6-013-2674-z

13. Javanshir N, Salehpour F, Aghazadeh J, Mirzaei F, Naseri Alavi SA (2017) The distribution of infection with Propionibacterium acnes is equal in patients with cervical and lumbar disc herniation. Eur Spine J 26:3135-3140. https://doi.org/10.1007/s0058 6-017-5219-z

14. Capoor MN, Ruzicka F, Schmitz JE, James GA, Machackova T, Jancalek R, Smrcka M, Lipina R, Ahmed FS, Alamin TF, Anand N, Baird JC, Bhatia N, Demir-Deviren S, Eastlack RK, Fisher S, Garfin SR, Gogia JS, Gokaslan ZL, Kuo CC, Lee Y-P, Mavrommatis K, Michu E, Noskova H, Raz A, Sana J, Shamie AN, Stewart PS, Stonemetz JL, Wang JC, Witham TF, Coscia MF, Birkenmaier C, Fischetti VA, Slaby O (2017) Propionibacterium acnes biofilm is present in intervertebral discs of patients undergoing microdiscectomy. PLOS ONE 12:e174518

15. Capoor MN, Ruzicka F, Machackova T, Jancalek R, Smrcka M, Schmitz JE, Hermanova M, Sana J, Michu E, Baird JC, Ahmed FS, Maca K, Lipina R, Alamin TF, Coscia MF, Stonemetz JL, Witham T, Ehrlich GD, Gokaslan ZL, Mavrommatis K, Birkenmaier C, Fischetti VA, Slaby O (2016) Prevalence of Propionibacterium acnes in intervertebral discs of patients undergoing lumbar microdiscectomy: a prospective cross-sectional study. PloS one 11:e0161676. https://doi.org/10.1371/journal.pone.0161676
16. Rao PJ, Phan K, Reddy R, Scherman DB, Taylor P, Mobbs RJ (2016) DISC (degenerate-disc infection study with contaminant control): pilot study of Australian cohort of patients without the contaminant control. Spine (Phila Pa 1976) 41:935-939. https:// doi.org/10.1097/brs.0000000000001404

17. Coscia MF, Denys GA, Wack MF (2016) Propionibacterium acnes, coagulase-negative staphylococcus, and the "Biofilm-like" Intervertebral Disc. SPINE 41:1860-1865

18. Achermann Y, Goldstein EJ, Coenye T, Shirtliff ME (2014) Propionibacterium acnes: from commensal to opportunistic biofilmassociated implant pathogen. Clin Microbiol Rev 27:419-440. https://doi.org/10.1128/cmr.00092-13

19. Perry A, Lambert P (2011) Propionibacterium acnes: infection beyond the skin. Expert Rev Anti Infect Ther 9:1149-1156. https ://doi.org/10.1586/eri.11.137

20. Albert HB, Kjaer P, Jensen TS, Sorensen JS, Bendix T, Manniche $\mathrm{C}$ (2008) Modic changes, possible causes and relation to low back pain. Med Hypotheses 70:361-368. https://doi.org/10.1016/j. mehy.2007.05.014

21. Li B, Dong Z, Wu Y, Zeng J, Zheng Q, Xiao B, Cai X, Xiao Z (2016) Association between lumbar disc degeneration and Propionibacterium acnes infection: clinical research and preliminary exploration of animal experiment. Spine (Phila Pa 1976) 41:E764-769. https://doi.org/10.1097/brs.0000000000001383

22. Dudli S, Liebenberg E, Magnitsky S, Miller S, Demir-Deviren S, Lotz JC (2016)Propionibacterium acnes infected intervertebral discs cause vertebralbone marrow lesions consistent with Modic changes. J Orthop Res 34:1447-1455. https://doi.org/10.1002/ jor.23265

23. Chen Z, Zheng Y, Yuan Y, Jiao Y, Xiao J, Zhou Z, Cao P (2016) Modic changes and disc degeneration caused by inoculation of Propionibacterium acnes inside intervertebral discs of rabbits: a pilot study. BioMed Res Int 2016:9612437. https://doi. org/10.1155/2016/9612437

24. Shan Z, Zhang X, Li S, Yu T, Liu J, Zhao F (2017) Propionibacterium acnes incubation in the discs can result in time-dependent Modic changes: a long-term rabbit model. Spine (Phila Pa 1976) 42:1595-1603. https://doi.org/10.1097/brs.0000000000002192

25. Zamora T, Palma J, Andia M, Garcia P, Wozniak A, Solar A, Campos M (2017) Effect of Propionibacterium acnes (PA) injection on intervertebral disc degeneration in a rat model: Does it mimic Modic changes? Orthop Traumatol Surg Res OTSR 103:795-799. https://doi.org/10.1016/j.otsr.2017.04.005

26. Ben-Galim P, Rand N, Giladi M, Schwartz D, Ashkenazi E, Millgram M, Dekel S, Floman Y (2006) Association between sciatica and microbial infection: true infection or culture contamination? Spine (Phila Pa 1976) 31:2507-2509

27. Carricajo A, Nuti C, Aubert E, Hatem O, Fonsale N, Mallaval FO, Vautrin AC, Brunon J, Aubert G (2007) Propionibacterium acnes contamination in lumbar disc surgery. J Hosp Infect 66:275-277

28. Lee MJ, Pottinger PS, Butler-Wu S, Bumgarner RE, Russ SM, Matsen FA 3rd (2014) Propionibacterium persists in the skin despite standard surgical preparation. J Bone Jt Surg 96:14471450. https://doi.org/10.2106/jbjs.m.01474

29. McLorinan GC, Glenn JV, McMullan MG, Patrick S (2005) Propionibacterium acnes wound contamination at the time of spinal surgery. Clin Orthop Relat Res 437(437):67-73

30. Chen Y, Wang X, Zhang X, Ren H, Huang B, Chen J, Liu J, Shan Z, Zhu Z, Zhao F (2018) Low virulence bacterial infections in cervical intervertebral discs: a prospective case series. Eur Spine J. https://doi.org/10.1007/s00586-018-5582-4

31. Hall GS, Pratt-Rippin K, Meisler DM, Washington JA, Roussel TJ, Miller D (2009) Growth curve for Propionibacterium acnes. Curr Eye Res 13:465-466. https://doi.org/10.3109/0271368940 8999875 
32. Butler-Wu SM, Burns EM, Pottinger PS, Magaret AS, Rakeman JL, Matsen IFA, Cookson BT (2011) Optimization of periprosthetic culture for diagnosis of Propionibacterium acnes prosthetic joint infection. J Clin Microbiol 49:2490-2495. https://doi. org/10.1128/jcm.00450-11

33. Arndt J, Charles YP, Koebel C, Bogorin I, Steib JP (2012) Bacteriology of degenerated lumbar intervertebral disks. J Spinal Disord Tech 25:E211-216. https://doi.org/10.1097/bsd.0b013e3182 69851a

34. Drago L, Romano CL, Cecchinato R, Villafane JH, De Vecchi E, Lamartina C, Berjano P (2017) Are MODIC Type 2 disc changes associated with low-grade infections? A pilot study. J Neurosurg Sci. https://doi.org/10.23736/S0390-5616.17.03997-2

35. Ohrt-Nissen S, Fritz BG, Walbom J, Kragh KN, Bjarnsholt T, Dahl B, Manniche C (2018) Bacterial biofilms: a possible mechanism for chronic infection in patients with lumbar disc herniation-a prospective proof-of-concept study using fluorescence in situ hybridization. APMIS : acta pathologica, microbiologica, et immunologica Scandinavica 126:440-447. https://doi. org/10.1111/apm.12841

36. Rajasekaran S, Tangavel C, Aiyer SN, Nayagam SM, Raveendran M, Demonte NL, Subbaiah P, Kanna R, Shetty AP, Dharmalingam K (2017) ISSLS PRIZE IN CLINICAL SCIENCE 2017: Is infection the possible initiator of disc disease? An insight from proteomic analysis. Eur Spine J 26:1384-1400. https://doi. org/10.1007/s00586-017-4972-3

37. Fritzell P, Bergstrom T, Welinder-Olsson C (2004) Detection of bacterial DNA in painful degenerated spinal discs in patients without signs of clinical infection. Eur Spine J 13:702-706

38. McDowell A, Al Perry, Lambert PA, Patrick S (2008) A new phylogenetic group of Propionibacterium acnes. J Med Microbiol $57: 218-224$
39. Urquhart DM, Zheng Y, Cheng AC, Rosenfeld JV, Chan P, Liew S, Hussain SM, Cicuttini FM (2015) Could low grade bacterial infection contribute to low back pain? A systematic review. BMC Med 13:13. https://doi.org/10.1186/s12916-015-0267-x

40. Ganko R, Rao PJ, Phan K, Mobbs RJ (2015) Can bacterial infection by low virulent organisms be a plausible cause for symptomatic disc degeneration? A systematic review. Spine 40:E587592. https://doi.org/10.1097/brs.0000000000000832

41. Dudli S, Miller S, Demir-Deviren S, Lotz JC (2017) Inflammatory response of disc cells against Propionibacterium acnes depends on the presence of lumbar Modic changes. Eur Spine J. https:// doi.org/10.1007/s00586-017-5291-4

42. Shan Z, Zhang X, Li S, Yu T, Mamuti M, Zhao F (2017) The influence of direct inoculation of Propionibacterium acnes on Modic changes in the spine: evidence from a rabbit model. J Bone Jt Surg 99:472-481. https://doi.org/10.2106/jbjs.16.00146

43. Magnitsky S, Dudli S, Tang X, Kaur J, Diaz J, Miller S, Lotz JC (2017) Quantification of propionic acid in the bovine spinal disk after infection of the tissue with $P$. Acnes bacteria. Spine. https:// doi.org/10.1097/brs.0000000000002448

44. Agarwal V, Golish SR, Alamin TF (2011) Bacteriologic culture of excised intervertebral disc from immunocompetent patients undergoing single level primary lumbar microdiscectomy. J Spinal Disord Tech 24(6):397-400

Publisher's Note Springer Nature remains neutral with regard to jurisdictional claims in published maps and institutional affiliations.

\section{Affiliations}

\section{Yucheng Jiao ${ }^{1,2} \cdot$ Yazhou Lin ${ }^{1,2} \cdot$ Yuehuan Zheng $^{3} \cdot$ Ye Yuan $^{4} \cdot$ Zhe Chen $^{1,2} \cdot$ Peng Cao ${ }^{1,2}$}

1 Department of Orthopedics, Ruijin Hospital, Shanghai Jiaotong University School of Medicine, 197 Ruijin Er Road, Shanghai 200025, China

2 Shanghai Key Laboratory for Prevention and Treatment of Bone and Joint Diseases with Integrated Chinese-Western Medicine, Shanghai Institute of Traumatology and Orthopedics, Ruijin Hospital, Shanghai Jiaotong University School of Medicine, Shanghai 200025, China
3 Department of Orthopedics, Ruijin Hospital North, Shanghai Jiaotong University School of Medicine, Shanghai 201800, China

4 Department of Orthopaedics, Chang Gung Hospital, Tsinghua University, Beijing 102202, China 